\title{
VICKREY-CLARKE-GROVES MECHANISMS \\ AND PERFECT COMPETITION
}

by

\author{
Louis Makowski \\ (UC Davis) \\ and \\ Joseph M. Ostroy \\ (UCLA)
}
UCLA Department of Economics
Working Paper \#333
July 1984

(Revised November 1985) 
Makowski, Louis and Ostroy, Joseph M. -- Vickrey-Clarke-Groves Mechanisms and Perfect Competition

We present a game theoretic-mechanism characterization of perfect competition: we prove the perfectly competitive mechanism is the only Pareto optimal, individually rational, dominant strategy allocation mechanism. Thus, perfect competition is uniquely capable of efficiently and non-coercively solving the incentive/bargaining problem when there is incomplete information, i.e., when each individual knows his own tastes and production possibilities better than anyone else. We also show one can interpret any dominant strategy, demand-revealing mechanism as "working" because it mimics the reward scheme that characterizes the perfectly competitive market, namely, the marginal product reward scheme. J. Econ. Theory,

(English). Makwoski: University of California, Davis; Ostroy: University of California, Los Angeles.

Journal of Economic Literature Classification Numbers $021,024,025,026$. 
I. Introduction

The goals of this paper are:

(i) to give a game theoretic-mechanism characterization of perfect competition, and

(ii) to provide an interpretation of dominant strategy, demand-revealing mechanisms as allocation schemes that mimic the perfectly competitive market.

The first goal is the primary focus of the formal part of the paper. The other involves an interpretation of the results and methods of proof used in achieving the first.

The traditional, partial-equilibrium description of a perfect competitor is someone whose purchase and sale decisions do not affect market price. We give a general equilibrium, mechanism version of the same idea. We define a class of economies as perfectly competitive if each member possesses a Walrasian equilibrium and if there is no change in any single individual's characteristics that would change Walrasian equilibrium prices.

Our definition of perfect competition is clearly stronger than the Walrasian definition. Accordingly, it can be used to make stronger assertions. In particular, a mechanism which yields Walrasian allocations is obviously both Pareto-optimal (PO) and individually rational (IR). If, in addition, no variation in an individual's characteristics leads to any change in prices, it readily follows that the mechanism exhibits the dominant strategy (DS) property. Thus if the class of possible economies is perfectly competitive then its Walrasian allocations are incentive compatible.

More interesting, and our main result, is that under standard convexity conditions the converse also holds: if a mechanism exhibits the PO, IR and DS properties, it must be a perfectly competitive mechanism. That is, it must yield Walrasian outcomes and the class of economies over which it is defined 
must be perfectly competitive. Thus, there is an equivalence between the contemporary concept of a mechanism with the DSPOIR properties and the traditional description of perfectly competitive economies as ones in which individuals cannot change prices. If and only if the class of possible economies is perfectly competitive is there no trade-off between efficiency and the dominant strategy version of incentive compatibility. This is our game theoretic-mechanism characterization of perfect competition. Notice our characterization of perfect competition is based on the dominant strategy standard for incentive compatibility. Thus, our result implies that perfect competition would be an incentive compatible way of realizing efficiency even in a world with incomplete information, where each individual knows his own tastes and production possibilities better than anyone else.

Because it requires that variations in an individual's demands have no effect on prices, the natural setting for a perfectly competitive economy contains a large number of participants. Roberts and Postlewaite [20] have demonstrated that with an increasingly large number of individuals a Walrasian equilibrium exhibits the dominant strategy property asymptotically. Others have demonstrated a converse. In particular, Hammond [7] provides conditions under which a symmetric DSPO mechanism must be Walrasian. The DS property is closely related to fair or no-envy allocations; Kleinberg [12], ChampsaurLaroque [1], McLennan [18] and Mas-Colell [17] have demonstrated that anonymous PO mechanisms must be Walrasian.

Our characterization of perfectly competitive economies (or equivalently, DSPOIR mechanisms) differs from these authors' results in that the former is based on an explicit definition of perfect competition. By contrast the above authors may be interpreted as seeking simultaneously (a) to ensure the 
existence of perfect competition by a large numbers argument and (b) to exposit the consequences of perfect competition. They thus combine the existence of perfect competition with its characterization. By contrast, working with an explicit definition allows us to completely sever the question of existence from that of characterization. It allows us to highlight-without obfuscating large numbers arguments--the consequences and special character of perfect competition; for example, the principle that the Walrasian mechanism is DSPOIR in--and only in--a class of perfectly competitive economies. Indeed, a useful result emerges from our approach: the above principle is independent of numbers. The current paper will establish the principle in a small numbers setting; a sequel will extend it to continuum economies and will contain some generic existence results. (Note: While the principles of perfect competition are independent of numbers, large numbers is essential for the generic existence of a DSPOIR mechanism. See Makowski and Ostroy [16]. But the theory is certainly not vacuous even with small numbers; Section 5 below contains an example.)

Staying within the small numbers setting, we can borrow from the dominant strategy mechanism literature: for example, Vickrey [22], Clarke [2], Groves [4], Groves and Loeb [6], Green and Laffont [3], Walker [23], and Holmström [8]. The family of allocation mechanisms studied in these papers we shall term Vickrey-Clarke-Groves (VCG) mechanisms. Our indebtedness to this literature, which will become transparent from the formal point of view, we nevertheless find surprising. After all, these contributions to mechanism theory were originally proposed to cope with allocational problems within a firm (Groves [4]) or with monopoly problems (Vickrey [22]) or public goods (Clarke [2], Groves and Loeb [6]) where the market does not operate or does not operate well. They take a non-market or unrelated-to-the-market approach 
that mechanism design can overcome incentive problems where markets fail. It is therefore surprising that the principles and mathematics behind these non-market allocation schemes should be so intimately related to the description of a perfectly competitive market. This is all the more so because we have come to expect that when there is a link between market and non-market allocation problems it is usually the other way around--the non-market problem is resolved with the help of market principles (e.g., the use of market-like internal pricing within the firm). This brings us to the second goal of the paper which is to argue that such an other-way-around interpretation is valid here, too.

How is such an other-way-around interpretation possible? We shall discover that there exists a common foundation to DS mechanisms and perfect competition. This common foundation is the concept of an individual's marginal product (MP), which we will see plays a crucial role both in our characterization of perfect competition and also in VCG mechanisms. This common foundation will lead us to argue that VCG mechanisms mimic, albeit imperfectly, the perfectly competitive market rather than the other way around.

The sequel is organized as follows. Section 2 presents our basic model, a standard demand-revealing model with quasi-linear preferences. Some of the fundamental results from mechanism theory that we shall utilize are displayed. Section 3 then proves, as a preliminary result, that any VCG mechanism is equivalent to what we call a MP mechanism; i.e., a mechanism that always gives each individual his/her MP, plus perhaps a lump sum. We then prove a main result of the paper. We prove the only VCG mechanism which is both feasible and non-coercive (IR) is what we call the full appropriation mechanism, i.e., the feasible mechanism that always gives each individual exactly his/her MP, 
without any lump sum. (We call it the full appropriation mechanism because under it each individual always fully appropriates his/her contribution to others, i.e, his/her MP.) This result we regard as a contribution to mechanism theory proper since it answers the mechanism-theoretic question: What is the class of feasible and non-coercive--hence "relevant"--VCG mechanisms? Section 4 relates this result to the market mechanism. It shows that the full appropriation mechanism is equivalent to the perfectly competitive mechanism. Hence, people receiving their MP's (in utility space) corresponds to perfect competition (in price space). When coupled with the main result of Section 3 this yields our game theoretic characterization of perfect competition as the unique DSPOIR allocation mechanism. Section 5 then illustrates our results with an example, and Section 6 concludes with some remarks that amplify on the import of the results in terms of our second goal.

\section{The Basic Model}

This section develops the basic model we shall be using, including the concept of a VCG mechanism and some of the fundamental results from mechanism theory.

We adopt a variant of the standard demand-revealing model applicable to private and/or public goods. There are $n$ individuals, indexed by $i$ and occasionally by $j$. The set of possible populations is $V \subset v_{1} x \ldots x V_{n}$, where $v_{j}$ represents the set of possible types for individual $i$. While it is common knowledge among individuals that they are in some population $v \in V$, each individual may only be sure of his/her own type, $v_{j}$. To exhibit a perfectly competitive example, in Section 4 below, it is important that $V$ can be a proper subset of the Cartesian product of the $v_{j}, i=1 \ldots n$.

There are $\ell+1$ commodities. The first $\ell$ we call " $y$-commodities", the last "money". Let $Y_{j} \subset R^{\ell}$ represent individual i's possible trades in the 
$y$-commodities. We assume for all $i$ that $Y_{j}$ is independent of $v_{j}$ and $0 \varepsilon Y_{i}$. The set of $y$-feasible outcomes for any entire population is defined by

$$
Y=\left\{y=\left(y_{1}, \ldots, y_{n}\right) \varepsilon \underset{i}{x} Y_{i}: \quad\left[y_{i}=0\right\} .\right.
$$

Adding money transfers to the model, let $x_{j}=\left(y_{i}, m_{i}\right) \varepsilon Y_{i} \times R$ represent $a$ possible trade for $i$ in both $y$-commodities and money. The set of feasible outcomes for the entire population is defined by

$$
X=\left\{x=(y, m) \varepsilon Y \times R^{n}: \text { each } x_{j}=\left(y_{i}, m_{j}\right) \text { and } \sum x_{j}=0\right\} \text {. }
$$

We assume individual i's actual type, $v_{j} \varepsilon V_{i}$, is completely described by his/her utility function over $y$-commodities; hence $v_{i}: Y_{j} \rightarrow R$. We also assume that $\forall i \forall v, v_{i}(\cdot)$ is continuous and $v_{j}(0)=0$. For any $v \in V$, the set of Y-efficient outcomes is represented by

$$
\operatorname{PO}(v)=\underset{\gamma}{\operatorname{argmax}} \sum v_{i}\left(y_{j}\right) \text {. }
$$

Each $i$ has a quasi-linear utility function over $y$-commodities and money of the form

$$
u_{i}\left(x_{i}, v_{i}\right)=v_{i}\left(y_{i}\right)+m_{i} .
$$

Thus, the set of Pareto optimal outcomes in $v$ is defined by

$$
P O(v)=\underset{X}{\operatorname{argmax}} \sum u_{i}\left(x_{j}, v_{j}\right) .
$$

Notice that quasi-linearity implies $x=(y, m) \in P O(v)$ iff $x \in X$ and $y \varepsilon P O Y(v)$. That is, the Pareto optimal outcomes in the model are simply the $Y$-efficient outcomes that are also feasible.

The total potential gains from trade in $v$ is given by

$$
g(v)=\sum u_{i}\left(x_{i}, v_{i}\right)=\sum v_{i}\left(y_{i}\right)
$$


for any $x=(y, m) \in P O(v)$.

Throughout $f: V \rightarrow Y \times R^{n}$ will denote a mechanism, where each $f(v)=(y(v), m(v))$. Interpret $f_{j}(v)=\left(y_{i}(v), m_{i}(v)\right)$ as the outcome to individual $i$ in population $v$. The mechanism $f$ is in the set of feasible mechanisms on $V, F[V]$, if $\forall v f(v) \varepsilon X$.

We say that $f \in D S P O Y[V]$, the set of dominant strategy, Y-efficient mechanisms on $v$, if $f(v)=(y(v), m(v))$ satisfies

$$
\begin{aligned}
& \forall v y(v) \in P O_{Y}(v) \\
& \forall v \nabla i, \text { if } v \mid v_{i}^{\prime} \equiv\left(v_{1}, \ldots, v_{i-1}, v_{i}^{\prime}, v_{i+1}, \ldots, v_{n}\right) \varepsilon v \text { then } \\
& \quad u_{i}\left(f_{i}(v), v_{i}\right) \geq u_{i}\left(f_{i}\left(v \mid v_{i}^{\prime}\right), v_{i}\right) .
\end{aligned}
$$

Notice if $V$ is smaller than the Cartesian product of the $V_{i}$ 's then (2) is a dominant strategy property only on the restricted domain $V$. Also notice $f \varepsilon D S P O Y[V]$ does not imply $f \varepsilon F[V]$.

The mechanism $f$ is in the family of Vickrey-Clarke-Groves mechanisms-sometimes more simply referred to as "Groves mechanisms"--on V, G[V], if it satisfies (1) and

$$
\forall v \forall i m_{i}(v)=\sum_{j \neq i} v_{j}\left(y_{j}\right)+h_{j}\left(v^{i}\right)
$$

where $v^{i} \varepsilon_{j \neq i} v_{j}$. Interpret $h_{j}\left(v^{i}\right)$ as a lump-sum transfer to $i$ since it is independent of $v_{\mathfrak{j}}$.

A basic result linking $G[V]$ with $D_{S P O Y}[V]$ uses the following definition. $v$ is smoothly connected means for any $v$ and $v \mid v_{i}^{\prime}$ in $v$, there exist $v \mid v_{j}^{\prime}(\alpha) \varepsilon v$ for $\alpha \in[0,1]$ satisfying:

$$
\begin{aligned}
& \text { (i) } v_{i}(0)=v_{i}, \\
& \text { (ii) } v_{i}(1)=v_{i}^{\prime} \text {, and }
\end{aligned}
$$


(iii) there is some number $K$ such that $\nabla \alpha \varepsilon[0,1], \nabla \beta \varepsilon[0,1]$, and

$$
\begin{aligned}
& \forall y_{i}=y_{i}\left(v \mid v_{j}(\beta)\right): \\
& \qquad\left|\frac{\partial v_{i}\left(y_{i}, \alpha\right)}{\partial \alpha}\right|<k<\infty .
\end{aligned}
$$

PROPOSITION 1A (Groves and Loeb [6]): G[V] C DSPOY[V].

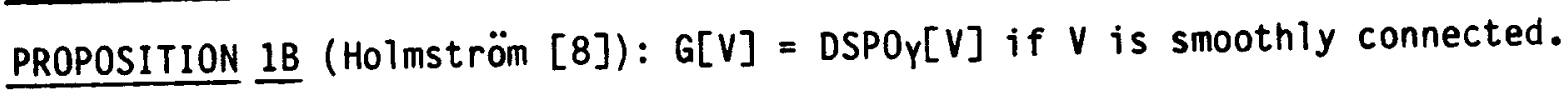

3. The Full Appropriation Mechanism: Incentive Compatibility without Prices

This section introduces the set of "full appropriation mechanisms" on $V$, and shows it is equivalent to the set of DSPOIR mechanisms on $V$.

To begin our study of the interplay between dominant strategy and perfectly competitive equilibria, it will be convenient to characterize VCG mechanisms as marginal product mechanisms. Apropos, let

$$
g^{i}(v)=\max _{Y} \sum_{j \neq i} v_{j}\left(y_{j}\right) \text { s.t. } y_{i}=0
$$

represent the maximum potential gains from trade in $v$ when $i$ does not participate. The marginal product of $i$ in $v$ is defined as

$$
\operatorname{MP}_{j}(v)=g(v)-g^{i}(v) \text {. }
$$

Notice $M P_{j}(v)$ equals i's total contribution to the potential gains from trade in $v$.

The mechanism $f$ is in the set of marginal product mechanisms on $V, M P[V]$, if it satisfies (1) and

$$
\forall v \forall i u_{j}\left(f_{j}(v), v_{j}\right)=M P_{j}(v)+\hat{h}_{j}\left(v^{i}\right)
$$

where $\hat{h}_{i}$ satisfies the same lump-sum restriction as $h_{j}$ in $(3)$. Denote by $\left.M \mathrm{MO}^{\mathrm{O}} \mathrm{V}\right]$ the set of marginal product mechanisms without lump sum transfers;

i.e., $f \varepsilon M P^{0}[V]$ means $f \varepsilon M P[V]$ and each function $\hat{h}_{i} \equiv 0$. 
As the following result confirms, it is easy to transform VCG mechanisms into MP mechanisms and vice-versa. So from now on we shall indicate mechanisms satisfying (1) and (3) or (1) and (4) as VCG-MP mechanisms.

THEOREM 1. $G[V]=M P[V]$

PROOF: If $f \in G[V]$, then since $m_{j}(v)=\sum_{j \neq j} v_{j}\left(y_{j}(v)\right)+h_{j}\left(v^{j}\right)$ and $u_{j}\left(f_{i}(v), v_{j}\right)=v_{j}\left(y_{i}(v)\right)+m_{j}(v)$, we have $u_{j}\left(f_{j}(v), v_{j}\right)=\sum_{j=1} v_{j}\left(y_{j}(v)\right)+h_{i}\left(v^{i}\right)$ $=g(v)+h_{j}\left(v^{i}\right)=\left[g(v)-g^{i}(v)\right]+\left[g^{i}(v)+h_{j}\left(v^{i}\right)\right] \equiv M P_{i}(v)+\hat{h}_{j}\left(v^{i}\right)$, using the fact that $g^{i}(v)$ does not depend on $v_{j}$. The converse just runs the argument in reverse.

The set of full appropriation mechanisms on $V, M P \star[V]$, is a particularly interesting subset of the VCG-MP family. A mechanism $f \varepsilon M P \star[V]$ iff, on all of V, it assigns a Pareto optimal (hence feasible) allocation that rewards each individual with exactly his/her marginal product. Hence,

$$
M P \star[V]=M P O[V] \cap F[V]
$$

In terms of the no surplus-marginal productivity theory of perfect competition (see 0stroy [19], Makowski [15]), f is a full appropriation mechanism iff in each population $v, f(v)$ is a "no surplus allocation". The intuition behind the "no surplus" terminology is as follows: $f \varepsilon M P *[V]$ means that in any population $v$, each individual fully appropriates his/her contribution to society's gains from trade; thus, he/she contributes "no surplus" to others in society.

Since $M P_{j}(v)$ is a unique number for each $i$ and $v$, all full appropriation mechanisms are utility equivalent: $f, f^{\prime} \varepsilon M P *[V]$ implies $u_{j}\left(f_{i}(v), v_{j}\right)=u_{i}\left(f_{j}^{\prime}(v), v_{j}\right)=M P_{j}(v)$ for all $i$ and $v$. Thus it is meaningful to speak about "the" full appropriation mechanism. 
The full appropriation mechanism differs from most members of the VCG-MP family in that it is always feasible and individually rational. A mechanism $f$ is individually rational on $V$, written $f \varepsilon I R[V]$, if

$\forall v \quad \nabla i u_{i}\left(f_{j}(v), v_{i}\right) \geq u_{i}\left(0, v_{j}\right)=0$.

THEOREM 2. $M P 0[V] \subset$ IR $[V]$.

PROOF: Let $f \varepsilon M^{0}[v]$. Then $\nabla v \nabla i u_{j}\left(f_{j}(v), v_{j}\right)=M P_{j}(v)=g(v)-g^{i}(v) \geq 0$ since $0 \in Y_{i} \cdot \|$

It follows that $M P \star[V] \subset I R[V]$.

With two mild qualifications, we show that the full appropriation mechanism is the only feasible and individually rational member of the VCG family. The qualifications are first discussed. When $M P_{j}(v)>0$, there is a complementarity (or gains from trade) between $v_{i}$ and $v^{i}$. Denote by $\underline{v}_{i}$ some type for $i$ satisfying $M P_{i}\left(v \mid \underline{v}_{i}\right)=0$. Thus $\underline{v}_{i}$ exhibits no complementarity with $v^{i}$.

In general, the difference between the sum of individual marginal products, $\sum M P_{j}(v)$, and the total gains from trade $g(v)$, can be positive or negative. However, in many well-known environments there are what we shall call non-decreasing returns to individuals: $\left[M P_{j}(v)-g(v) \geq 0\right.$. This follows the terminology in production theory which says that under nondecreasing returns to scale the sum of the payments to the factors of production according to their marginal products is at least as large as the total product. We now verify the principal claim of this section.

\section{THEOREM 3. Suppose} $\forall v \forall i$ there exists some population $v \mid \underline{v}_{i} \varepsilon V$ such that $\operatorname{MP}_{i}\left(v \mid \underline{v}_{i}\right)=0$.

Then $M P *[V]=G[V] \cap F[V] \cap I R[V]$. 
PROOF: $f \varepsilon G[V] \cap F[V]$ implies $f \in M P[V] \cap F[V]$. Hence, since $\forall v f(v) \varepsilon P 0(v)$, $g(v)=\sum u_{j}\left(f_{j}(v), v_{j}\right)=\left[\left[M P_{j}(v)+\hat{h}_{j}\left(v^{i}\right)\right]\right.$. This shows using (7) that

$0 \geq \sum \hat{h}_{i}\left(v^{i}\right)$. But if $f \varepsilon \operatorname{IR}[V]$ and $(6)$ holds then $u_{j}\left(f_{i}\left(v \mid \underline{v}_{i}\right), \underline{v}_{i}\right)=M P_{j}\left(v \mid \underline{v}_{j}\right)+$ $\hat{h}_{j}\left(v^{i}\right)=0+\hat{h}_{j}\left(v^{i}\right) \geq 0$ for each $i$. Thus, $\nabla v \nabla i \hat{h}_{j}\left(v^{i}\right)=0$.

To show that non-decreasing returns is characteristic of many economic models, define the "game" (in characteristic function form) associated with the population $v$ by

$$
g_{v}(S)=\max _{Y}\left\{\sum v_{j}\left(y_{i}\right): y_{i}=0, i t s\right\}
$$

for all $S \subset A \equiv\{1, \ldots, n\}$. Let $I_{S}$ be the characteristic function of $S$ regarded as an element of $R^{n}$. The game $g_{V}$ is balanced if for any collection $\{S\}$ with $\lambda_{S}>0$ and $\sum \lambda_{S} l_{S}=1_{A}, \sum \lambda_{S} g_{V}(S) \leq g_{V}(A)$. For games with transferable utility, Shapley [1967] has demonstrated that a game is balanced if and only if it has a non-empty core, which in our terms is an $x \varepsilon X$ such that for all $S, \sum_{i \in S} u_{i}\left(x_{j}, v_{j}\right) \geq g_{v}(S)$.

THEOREM 4. Suppose for all $\vee \varepsilon \vee g_{V}$ is balanced or, equivalently, $v$ has a non-empty core. Then there are non-decreasing returns to individuals, (7).

PROOF: Let $x$ be in the core of $v$. Then for each $i g_{v}(A \backslash\{i\}) \leq \sum_{j \neq i} u_{j}\left(x_{j}, v_{j}\right)$ and $g_{v}(A)=\sum_{j} u_{j}\left(x_{j}, v_{j}\right)$. Hence for each $i M P_{j}(v)=g(v)-g^{i}(v)=g_{v}(A)-$ $g_{v}(A \backslash\{i\}) \geq u_{j}\left(x_{i}, v_{j}\right)$. Summing over $i$ shows $\sum M_{i}(v) \geq \sum u_{j}\left(x_{j}, v_{j}\right)=g_{v}(A)=$ $g(v)$.

It is interesting to observe that if (7) holds then $x \in X$ and $u_{j}\left(x_{j}, v_{j}\right)=$ $M P_{j}(v)$ for all $i$ implies $x \in P O(v)$. That is, any feasible allocation that gives everyone exactly their marginal product is Pareto optimal. (Proof: 
$\sum u_{j}\left(x_{i}, v_{j}\right)=\sum M P_{j}(v) \geq g(v)$, and $>$ implies $\left.x \in x_{0}\right)$ Hence, with (7), $f \varepsilon M P[V] \cap F[V]$ iff $f$ is a feasible mapping that always gives everyone exactly their marginal product.

Let us summarize the results of our findings. If $V$ is smoothly connected, if each $\vee \in V$ has a non-empty core, and if $V$ is sufficiently large always to permit anyone to claim characteristics exhibiting no compelementarity with others, then the full appropriation mechanism is the only Pareto-efficient, individually rational, dominant strategy mechanism.

REMARK: (The relation between the MP and other demand-revealing mechanisms.) $M P O$ is identical to the demand-revealing mechanism for private goods proposed by Vickrey [22]. Our assumption (7) implies $\sum m_{i}(v)=$ $\left[M P_{j}(v)-g(v) \geq 0\right.$, so the $M P 0$ mechanism runs a deficit whenever it is not feasible. Other versions of demand-revealing mechanisms are designed to yield, whenever $\left[m_{i}(v) \neq 0\right.$, a surplus. See Clarke [2] and Groves and Loeb [6] for public goods. Loeb [14] presents a common framework for describing demand-revealing mechanisms yielding a surplus or deficit and including public or private goods. Similarly, ours is intended as a framework for public or private goods.

In the references above, the technologies typically satisfy (6) and (7), needed for Theorem 3. But there is another much-studied class of technologies in the demand-revealing literature (see, for example, Green and Laffont [3]) in which a variant of our MPO plays a prominent role, but for which conditions (6) and (7) may fail. We shall outline here an argument leading to the conclusion that the singular properties of $M P^{0}$, when $\left[m_{i}(v)=0\right.$, described in Theorem 3 also continue to hold in these technologies, despite the absence of (6) and (7). 
Let the technology $Y$ be a fixed set of costless, alternative public projects and $v_{j}: Y+R$. Normalize tastes so that for some yozY, $v_{j}\left(y_{0}\right)=0 \nabla v \nabla i$. Now redefine $M P_{i}(v) \equiv g(v)-g\left(v \mid v_{j}{ }^{0}\right)$, where $g(v) \equiv \max _{\gamma}\left[v_{j}(y)\right.$ and $v_{j}{ }^{0}$ is the null function on $Y$. Thus $g\left(v \mid v_{j}{ }^{0}\right)$ plays the role of $g^{i}(v)$ in the sense that it measures the maximum gains in the economy when $i$ is effectively absent. Under these definitions of the technology and i's marginal product, MP0 is commonly called the pivot mechanism: $\forall i m_{j}(v)=\sum_{j \neq i} v_{j}(y(v))-g\left(v \mid v_{i}{ }^{0}\right)$.

Unlike other public good models, in this one there is always non-increasing returns to individuals: $\forall v \quad \sum M P_{i}(v)-g(v) \leq 0$

This is because the production of the public good is assumed to be costless, eliminating the cost-sharing advantages in the production of public goods. (Note: if exclusion were possible, it would follow from Theorem 4 that the core of such an economy would typically be empty.)

Replace hypothesis (6) with the condition that $\forall v \quad \forall i$ there exists some population $v \mid \underline{v}_{i} \in V$ such that $g\left(v \mid \underline{v}_{i}\right)=0$. According to $\left(6^{\prime}\right)$ individual i can always exhibit preferences for the alternatives in $Y$ making the "status quo" alternative yo a Pareto optimum. It readily follows (see Laffont and Maskin [13]) that under hypotheses (6') and (7') the conclusions of Theorem 3 continue to hold. Therefore, when $v$ is smoothly connected, the full appropriation mechanism is the only DSPOIR mechanism.

4. The Perfectly Competitive Mechanism: Incentive Compatibility with Prices This section introduces the "perfectly competitive mechanism" on $V$, and shows it is equivalent--under convexity conditions--to the full appropriation 
mechanism on $V$.

In partial equilibrium theory, a perfect competitor is one who cannot influence prices. In Walrasian general equilibrium the absence of influence is taken for granted, to focus on the problem of simultaneous equilibrium in many markets. We shall combine the partial and general equilibrium approaches to define a perfectly competitive mechanism: a Walrasian mechanism that explicitly incorporates the partial equilibrium notion of a perfect competitor.

First, define the better than $x_{i}$ set for type $v_{i}$ as

$$
B_{j}\left(x_{i}, v_{j}\right)=\left\{x_{i}^{\prime} \varepsilon Y_{i} \times R: u_{j}\left(x_{i}^{\prime}, v_{j}\right)>u_{i}\left(x_{i}, v_{i}\right)\right\}
$$

A Walrasian equilibrium for $v$ is an $(x, p) \varepsilon X \times R^{\ell+1}$ such that $\forall i, p x_{i}=0<$ $\mathrm{PB}_{j}\left(x_{j}, v_{j}\right)$. The set of such pairs will be denoted by $W(v)$.

Say that $f$ is in the set of perfectly competitive mechanisms on $V$, $P C[V]$, if

$$
\forall v(f(v), p(v)) \varepsilon W(v) \text { for some } p(v) \varepsilon R^{l+1} \text {, and }
$$$$
\forall v \forall i \quad \forall v \mid v_{j}^{\prime} \in v\left(f\left(v \mid v_{i}^{\prime}\right), p(v)\right) \in W\left(v \mid v_{j}^{\prime}\right) \text {. }
$$

Thus any individual $i$, no matter what his/her characteristics in $V$, cannot influence Walrasian prices. Alternatively, a perfectly competitive mechanism is one in which individuals face perfectly elastic demands and supplies at market-clearing Walrasian prices. Because a Walrasian mechanism is generally manipulable, (8a) by itself does not imply (8b).

The following result, when coupled with its converse to be developed next, yields a price-theoretic version of Theorem 3 .

THEOREM 5A. PC $[V] \subset D S P O Y[V] \cap F[V] \cap I R[V] \equiv D S P O I R[V]$.

THEOREM 5B. Assuming (6), PC[V] CMP* $[V]$. 
Theorem $5 \mathrm{~A}$ says that when individuals cannot influence prices in Walrasian equilibrium, the associated mechanism satisfies the dominant strategy property. (This formally proves the rather obvious fact that a dominant strategy property underlies the Marshallian definition of perfect competition.) Theorem 5B says that if $V$ is sufficiently large always to permit anyone to claim characteristics exhibiting no complementarity with others, then any perfectly competitive mechanism must involve full appropriation: each individual must always receive his/her marginal product.

PROOF OF 5A. $f \varepsilon P C[V]$ is well-known to imply $f \varepsilon F[V] \cap P O \gamma[V] \cap$ IR[V]. To show $f \in D S[V]$, simply observe that if $(f(v), p(v)) \varepsilon W(v)$ and $p(v) f_{j}(v)=$ $p(v) f_{i}\left(v \mid v_{j}^{\prime}\right)=0$ for each $v \mid v_{j}^{\prime} \varepsilon v$, then $u_{j}\left(f_{i}(v), v_{j}\right) \geq u_{j}\left(f_{j}\left(v \mid v_{j}^{\prime}\right), v_{j}\right)$. \| PROOF OF 5B. We need to verify $\nabla v \nabla i u_{j}\left(f_{j}(v), v_{j}\right)=M P_{j}(v)$. But $M P_{j}(v) \equiv$ $g(v)-g^{i}(v)=g(v)-g\left(v \mid \underline{v}_{i}\right)[$ by $(6)]=\sum_{j} u_{j}\left(f_{j}(v), v_{j}\right)-\left[\sum_{j \neq j} u_{j}\left(f_{j}\left(v \mid \underline{v}_{j}\right), v_{j}\right)+\right.$ $\left.u_{j}\left(f_{j}\left(v \mid \underline{v}_{i}\right), \underline{v}_{i}\right)\right]$ since any $W(v)$ is efficient. So $M P_{i}(v)=u_{j}\left(f_{j}(v), v_{j}\right)-$ $u_{i}\left(f_{i}\left(v \mid \underline{v}_{i}\right), \underline{v}_{i}\right)$ since $\nabla j \neq i u_{j}\left(f_{j}(v), v_{j}\right)=u_{j}\left(f_{j}\left(v \mid \underline{v}_{i}\right), v_{j}\right)$ using the fact that both $f(v)$ and $f\left(v \mid \underline{v}_{i}\right)$ are supported by $p(v)$.

To complete the proof we need only verify $u_{j}\left(f_{i}\left(v \mid \underline{v}_{j}\right), \underline{v}_{i}\right)=0$. But $\sum_{j \neq i} u_{j}\left(f_{j}\left(v \mid \underline{v}_{j}\right), v_{j}\right) \geq g^{j}(v)$ since any $W(v)$ is in the core. So, since $\sum_{j \neq j}^{j \neq i} u_{j}\left(f_{j}\left(v \mid \underline{v}_{i}\right), v_{j}\right)+u_{i}\left(f_{j}\left(v \mid \underline{v}_{i}\right), \underline{v}_{j}\right)=g\left(v \mid \underline{v}_{i}\right)=g^{i}(v), u_{j}\left(f_{j}\left(v \mid \underline{v}_{i}\right), \underline{v}_{j}\right) \leq 0$. But $\left(f\left(v \mid \underline{v}_{i}\right), p(v)\right) \varepsilon W\left(v \mid \underline{v}_{j}\right)$ implies $u_{j}\left(f_{i}\left(v \mid \underline{v}_{j}\right), \underline{v}_{i}\right) \geq 0$ since $0 \varepsilon Y_{i}$. The desired conclusion follows.

We conclude this section with a converse to Theorem 5B. This will require two additional assumptions. Let

$$
B_{v}(\alpha) \equiv\left\{\sum x_{i} \varepsilon \sum Y_{i} \times R: \sum u_{j}\left(x_{i}, v_{i}\right)>\alpha\right\}
$$

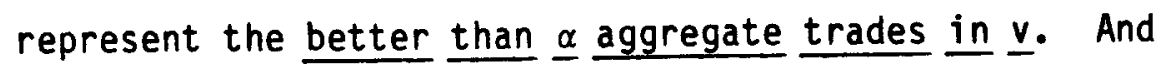




$$
B_{v}^{i}(\alpha) \equiv\left\{\sum_{j \neq i} X_{j} \in \sum_{j \neq i} Y_{j} \times R: \sum_{j \neq i} u_{j}\left(x_{j}, v_{j}\right)>\alpha\right\}
$$

represent the better than $\underline{\alpha} \underline{\text { aggregate }} \underline{\text { trades }}$ in $\underline{v}$, without $\underline{i}$. Notice by . construction $0 \notin B_{v}(g(v))$ and $0 \& B_{v}^{j}\left(g^{i}(v)\right)$. The first assumption is that all Pareto-optimal allocations can be supported by efficiency prices:

$$
\forall v \exists p(v) \in R^{l+1} \text { such that } p(v) B_{v}(g(v))>0 \text {. }
$$

If, for example, each $Y_{j}$ is convex, each utility function $v_{i}$ is quasi-concave, and any $x \in P O(v)$ has the property that $\left[x_{i} \varepsilon\right.$ interior $\left\{\left[Y_{j}\right\}\right.$, then (9) can be derived using standard arguments.

The second assumption is that

$$
\begin{aligned}
& \nabla v \nabla i \exists p^{i}(v) \in R^{\ell+1} \text { such that } p^{i}(v) B_{v}^{i}\left(g^{i}(v)\right)>0 . \\
& \text { Further, if } p B_{v}^{i}\left(g^{i}(v)\right)>0 \text { then for some } \lambda>0, p=\lambda p^{i}(v) \text {. }
\end{aligned}
$$

Condition (10) says that not only can zero be separated from $\operatorname{Bv}_{v}^{i}\left(g^{i}(v)\right)$ by a hyperplane, but also there is only one such separating hyperplane. The uniqueness part of (10) would be satisfied, for example, if any $j \neq i$ has a smooth indifference curve through $x_{j}$, where $x_{j}$ is part of any $x \in X$ satisfying $g^{i}(v)=\sum_{j \neq i} u_{j}\left(x_{j}, v_{j}\right)$ and $x_{j}=0$.

THEOREM 6A. Assume $(9)$ and suppose $f \varepsilon M P *[v]$. Then $\nabla v] p(v)$ such that $(f(v), p(v)) \varepsilon W(v)$. Further, $\nabla v \nabla i \nabla(x, p) \varepsilon W(v), u_{i}\left(x_{i}, v_{j}\right)=u_{i}\left(f_{i}(v), v_{j}\right)=M P_{j}(v)$. THEOREM 6B. Assume $(9)$ and $(10)$. Then $M P *[V] \subset P C(V]$.

An immediate implication of Theorem $6 \mathrm{~A}$ is that condition (9) implies the nondecreasing-returns condition (7). This follows from Theorem 4 since Walrasian allocations are in the core. Notice the second assertion of Theorem 6A says all Walrasian allocations are utility equivalent; it will be useful for proving Proposition 3 in Section 5 below. 
To summarize, we have demonstrated that if $V$ satisfies conditions (6), (9), (10), and is smoothly connected, the following are equivalent characterizations of the full appropriation mechanism:

(i) It is the only feasible mechanism that always rewards each individual with exactly his/her marginal product.

(ii) It is the only Pareto optimal, individually rational, dominant strategy mechanism.

(iii) It is the only perfectly competitive mechanism.

PROOF OF $6 A$ : Since $\sum u_{i}\left(f_{i}(v), v_{i}\right)=g(v), \sum B_{i}\left(f_{j}(v), v_{j}\right)=B_{v}(g(v))$.

Therefore, by (9) $] p \in R^{l+1}$ such that $p \sum B_{j}\left(f_{j}(v), v_{j}\right)>0$. Since $\left[f_{j}(v)=\right.$ 0 , it readily follows that $p f_{j}(v)<p B_{j}\left(f_{i}(v), v_{j}\right)$ for all $i$. To show that $(f(v), p) \in W(v)$, it suffices to show that $p f_{i}(v)=0$ for all $i$.

$$
\text { For } \forall i, u_{i}\left(f_{i}(v), v_{j}\right)=g(v)-g^{i}(v) \text { implies } g^{i}(v)=\sum_{j \neq j} u_{j}\left(f_{j}(v), v_{j}\right) \text {. }
$$

Let $x^{i}(v) \varepsilon x$ be such that $x_{j}^{j}(v)=0$ and $\sum_{j \neq i} u_{j}\left(x_{j}^{j}(v), v_{j}\right) \stackrel{j \neq j}{=} g^{i}(v)$. Since $g^{i}(v)=$ $\sum_{j \neq j} u_{j}\left(f_{j}(v), v_{j}\right)$, the money components of the $x_{j}^{j}(v)$ can be adjusted to ensure $u_{j}\left(x_{j}^{j}(v), v_{j}\right)=u_{j}\left(f_{j}(v), v_{j}\right)$ for each $j$. Therefore, $\forall j \neq i, p x_{j}^{j}(v) \geq p f_{j}(v)$. Since $\sum_{j \neq j} x_{j}^{i}(v)=0$, we conclude that $0 \geq p\left(\sum_{j \neq i} f_{j}(v)\right)=-p f_{i}(v)$. Thus, $\nabla i f_{i}(v) \geq 0$. But $\left[f_{j}(v)=0\right.$, so the desired conclusion follows.

To show all Walrasian allocations are utility equivalent, suppose $(x, p) \varepsilon$ $W(v)$ and there is an $i$ such that $\sum_{j \neq i} u_{j}\left(x_{j}, v_{j}\right)<\sum_{j \neq i} u_{j}\left(f_{j}(v), v_{j}\right)=g^{i}(v)$. This contradicts all Walrasian allocations are in the core. So, $\nabla i$

$\sum_{j \neq i} u_{j}\left(x_{j}, v_{j}\right) \geq \sum_{j \neq i} u_{j}\left(f_{j}(v), v_{j}\right)$, which implies $\forall i u_{j}\left(x_{j}, v_{j}\right) \leq u_{i}\left(f_{j}(v), v_{j}\right)$ since $\sum_{j}^{j \neq i} u_{i}\left(x_{j}, v_{j}\right)=\sum_{i} u_{j}\left(f_{j}(v), v_{j}\right)=g(v)$. So, $v i u_{j}\left(x_{j}, v_{j}\right)=u_{i}\left(f_{j}(v), v_{j}\right)$ since otherwise $\sum_{i} u_{i}\left(x_{i}, v_{i}\right)<\sum_{i} u_{i}\left(f_{i}(v), v_{i}\right)=g(v)$.

PRO0F OF $6 \mathrm{~B}$ : By $6 A, \nabla v j p(v)$ such that $(f(v), p(v) \in W(v)$. Therefore $p(v)\left[\sum_{j \neq i} B_{j}\left(f_{j}(v), v_{j}\right)\right]>0$, or since $\sum_{j \neq i} u_{j}\left(f_{j}(v), v_{j}\right)=g^{i}(v), p(v) B_{v}^{i}\left(g^{i}(v)\right)>0$. Again by $6 A, \forall v \mid v_{j}^{\prime} \exists p\left(v \mid v_{j}^{\prime}\right)$ such that $\left(f\left(v \mid v_{j}^{\prime}\right), p\left(v \mid v_{j}^{\prime}\right)\right) \varepsilon W\left(v \mid v_{j}^{\prime}\right)$. So, 
since $\sum_{j \neq j} u_{j}\left(f\left(v \mid v_{j}^{\prime}\right), v_{j}\right)=g^{i}\left(v \mid v_{j}^{\prime}\right), p\left(v \mid v_{j}^{j}\right) B_{v}^{i} v_{i}\left(g^{i}\left(v \mid v_{j}^{\prime}\right)\right)>0$. But by construction $g^{i}(v)=g^{i}\left(v \mid v_{j}^{\prime}\right)$. Therefore, by $(10), p\left(v \mid v_{j}^{\prime}\right)=\lambda p(v)$ for some $\lambda>0$. This implies $\left(f\left(v \mid v_{j}^{\prime}\right), p(v)\right) \in W\left(v \mid v_{j}^{\prime}\right)$.

5. The Existence of Perfectly Competitive Environments: A Private Good Example Define a perfectly competitive environment as an environment $(v, X)--i . e$., a population plus a set of feasible outcomes--in which it is feasible for every individual to receive his/her MP. We know the full appropriation mechanism can operate on any family $(V, X)$ of such environments. This section shows, by way of a simple private good example, that families of such environments exist even in finite economies. 1

Consider a family of environments defined by the following: (i) $l=1$; (ii) the $n$ individuals are partitioned into a set $B$ of buyers and a set $S$ of sellers such that $Y_{i}=[0,1]$ if $i \varepsilon B$ and $Y_{i}=[-1,0]$ if $i \varepsilon S$; (iii) $\forall i V_{i}=$ $\left\{v_{j}: \quad v_{j}\left(y_{i}\right)=w_{j} y_{j}\right.$ for all $y_{j} \varepsilon Y_{j}$ and some $\left.w_{j} \varepsilon R_{+}\right\}$; so $u_{j}\left(x_{j}, v_{j}\right)=w_{j} y_{j}+m_{j}$. Thus each $i$ enjoys constant marginal utility from the $y$-commodity if $i \varepsilon B$, suffers constant marginal cost if $i \varepsilon S$, and no individual can trade more than one unit of $y$.

Let $V=V_{1} \times \ldots \times V_{n}$. Since each type $v_{j} \varepsilon V_{j}$ is uniquely identified by a scalar parameter, namely a $w_{j} \varepsilon R_{+}$, we may identify the set $\nabla$ with $R_{+}^{n} \cdot A$ description of any $v \varepsilon \hat{V}$ along with its Walrasian equilibrium can be $i l l u s t r a t e d$ in a demand-and-supply diagram. Within $\nabla$ we highlight those populations for which there is a unique Walrasian equilibrium price, when the price of money is normalized to unity. Call this set $V^{*} . A \vee \varepsilon V^{*}$ is depicted in Figure 1 and a $v \varepsilon \nabla \backslash v^{*}$ in Figure 2 . 


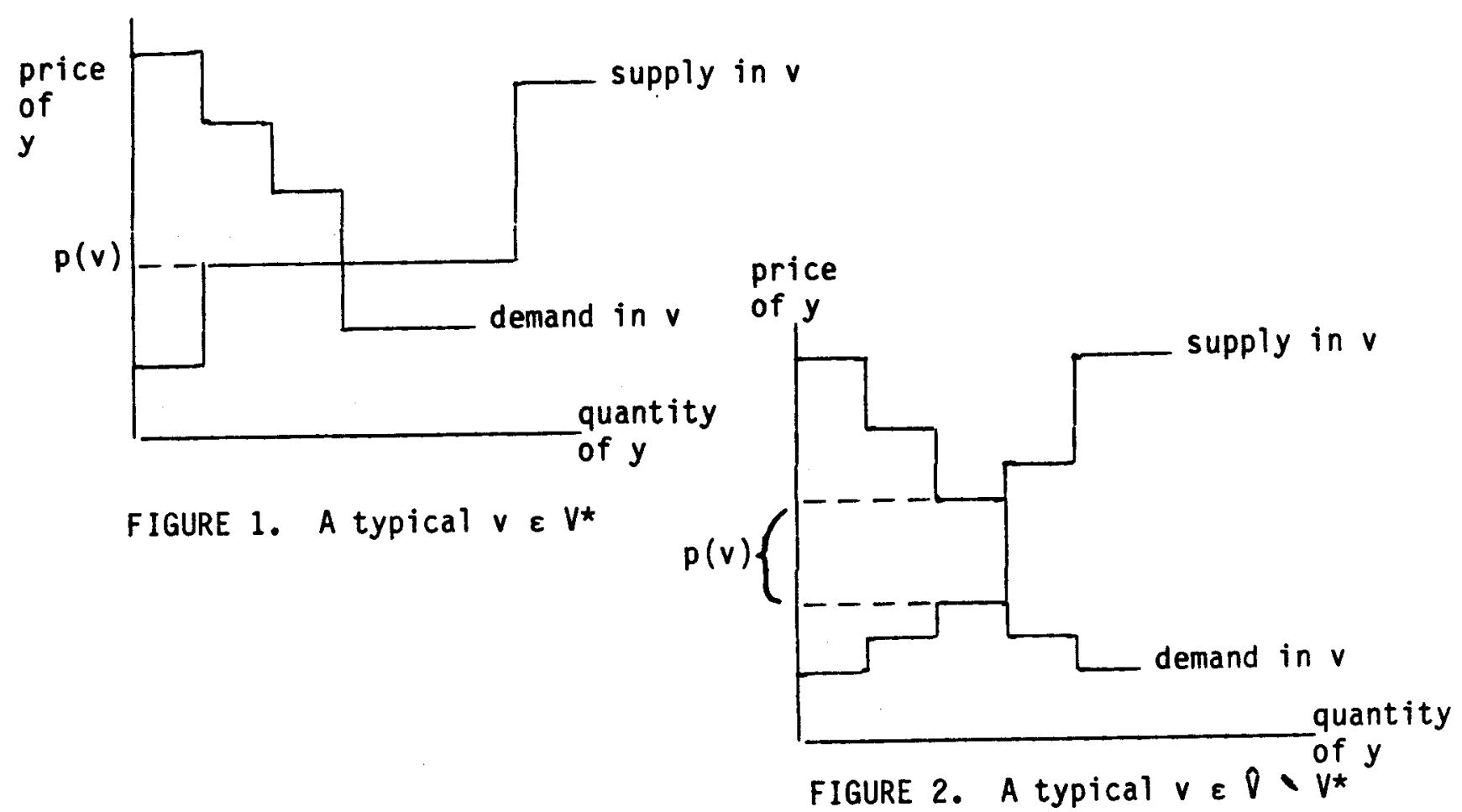

Inspection of Figure 1 reveals that because $Y_{i}=[0,1]$ or $[-1,0]$, no individual can change $p(v)$ by changing to some $v_{i}^{\prime}$ as long as both $v$ and $v \mid v_{i}^{\prime}$ belong to $v^{*}$. If $v \mid v_{i}^{\prime} \varepsilon v^{*}$, then $i$ just moves the demand or supply in $v$ over by "one step" without changing $p(v)$. If $v \mid v_{i}^{\prime}$ moves the demand and supply schedules so that there are multiple price equilibria (as in Figure 2), then $v \mid v_{j}^{\prime} t v^{*}$. This leads to the following:

PROPOSITION 2: Let $f^{*}: v^{*} \rightarrow X$ satisfy for each $v\left(f^{*}(v), p(v)\right) \varepsilon W(v)$. Then $f * \varepsilon M P *[V *]=\operatorname{DSPOIR}[V *]=\operatorname{PC}[V \star]$.

PROOF: The discussion above says that any Walrasian mechanism on $V *$ is in $P C\left[V^{\star}\right]$. This implies $f^{\star} \in P C\left[V^{\star}\right]$. It also implies $M P \star\left[V^{\star}\right] \subset P C\left[V^{\star}\right]$ since by Theorem $6 \mathrm{~A}$ any full appropriation mechanism on $V^{*}$ is a Walrasian mechanism. Conversely, $M P \star\left[V^{\star}\right] \supset P C\left[V^{*}\right]$ by Theorem $5 B$. Hence $M P \star\left[V^{\star}\right]=P C\left[V^{\star}\right]$. Notice by letting $\underline{w}_{i}=p(v)$ one verifies $V^{*}$ is sufficiently large to admit the possibility of no-complementarity, (6). 
It is readily verified that $V$ * is smoothly connected. In particular, if $v$ and $v \mid v_{i}^{\prime} \varepsilon v^{*}$ so is $v \mid v_{i}^{\prime}(\alpha)$ where $v_{j}(\alpha)=\alpha v_{i}+(1-\alpha) v_{i}^{\prime}$. Also, since $W(v) \neq \phi$, there are non-decreasing returns, (7). Therefore, by Proposition $1 B$ and Theorem 3, MP* $[V \star]=\operatorname{DSPOIR}[V \star] .2$

Given the negative conclusions of Hurwicz [9] for DSPOIR mechanisms and of Walker [24] even for DSPO mechanisms, the existence of the above $V$ * is somewhat surprising. Of course, $V \star$ was chosen carefully. Note for example that while it is a subset of $O, V^{\star}$ does not form a Cartesian product. Using a somewhat different class of utility functions, Uzi Segal has found a family of perfectly competitive environments that is a Cartesian product.

When the domain is all of $D$, we can obtain an independent proof of the non-existence of a perfectly competitive mechanism.

PROPOSITION 3: $\quad M P *[O]=$ DSPOIR $[O]=P C[O]=\phi$.

PROOF: $\quad \nabla$ is smoothly connected and satisfies $(6)$. Since $\forall v W(v) \neq \phi$, it also satisfies (7). Thus the first equality follows from Proposition $1 B$ and Theorem 3. Since Theorem $5 B$ implies $P C[\hat{V}] \subset M P \star[\hat{V}]$, it suffices to show $M P *[\hat{V}]=\phi$. If the contrary then by Theorem $6 \mathrm{~A}$, for all $v \varepsilon \hat{V} W(v)$ consists of utility equivalent allocations. Consider $v \in D \backslash V *$. Then Walrasian prices vary within $W(v)$. So whenever $g(v)>0, W(v)$ consists of utility non-equivalent allocations. "

Proposition 3 demonstrates that even in this most elementary class of market environments, the negative conclusions on incentives for preferencerevelation first pointed out by Hurwicz [1972] also obtain. To restate the results of this paper, such negative conclusions on the existence of efficient, individually rational, dominant strategy mechanisms are simply a reflection of the fact that the environments considered are imperfectly competitive. If and only if one is in a perfectly competitive environment do such mechanisms exist. 
6. Conclusion

In Section 4 we proved that the feasible mechanism which always rewards everyone with exactly his/her MP--the full appropriation mechanism--is equivalent to the perfectly competitive mechanism. Thus we proved that rewarding people with their MP's--full appropriation--is the abstract, non-price principle underlying perfectly competitive markets. On this basis, we take it for granted that any mechanism that works because it involves a MP reward scheme is based on perfectly competitive market principles.

This train of thought leads us to view all Groves mechanisms as trying to mimic the perfectly competitive market. To see why, recall in Section 3 we proved all such schemes may be viewed as rewarding everyone with his/her MP, plus perhaps a lump sum. (Recall lump sums do not affect incentives.) of course, Groves schemes can be defined over classes of environments in which full appropriation is impossible--the sum of people's MP's just does not add up to the total possible gains from trade. Based on the above, we interpret Groves schemes on such more general environments as an attempt to restructure incentives so as to make the allocation problem obey the MP reward principle underlying perfectly competitive markets. But, as we know, in such environments no Groves scheme will generally be feasible. Their failure is reflected in the scheme's budgetary surplus or deficit (Walker [24], Hurwicz and Walker [11]). We interpret this failure as reflecting the fact that the class of environments is inconsistent with full appropriation, i.e., fails to be perfectly competitive. It is along these lines that we interpret Groves-DS mechanisms as mimicking, albeit imperfectly, the perfectly competitive market rather than the other way around.

It is interesting to notice that an arbitrary Walrasian equilibrium need not involve full appropriation: The MP reward principle characterizes a 
perfectly competitive market, but not necessarily an arbitrary Walrasian market. Accordingly, price-taking in the latter need not be incentive compatible. The remarkable incentive effect of a MP reward system--that it gives the incentives for realizing an efficient outcome--has long been appreciated (more or less) in the oral tradition of economic theory. But this incentive effect was largely lost sight of in the heyday of incentive-free thinking about the market in terms of the Walrasian model. Only when economists' interest in solving incentive problems became renewed was the potency of the MP reward principle rediscovered, in the form of Groves mechanisms. Indeed, the tools and main results of mechanism theory allow for a precise, modern-day expression of its potency. So, we conclude with a historical paradox. Far from showing how a purposefully designed mechanism can do what competitive markets cannot, mechanism theory, by posing and solving the dominant strategy or revelation problem, has rediscovered and given a new articulation to the under-lying logic of competitive market equilibrium! That is, it has rediscovered and rearticulated the importance of rewarding people with their MP's. 


\section{Footnotes}

1. A parallel, public good example of the model is available in an earlier version of the paper.

2. Although the uniqueness part of $(10)$ isn't satisfied by all veV*, notice the conclusion of Theorem 6B still holds on $V *$. This shows (10) is sufficient but not necessary for the conclusion. A subset of $V *$ will satisfy (10). Namely, the set of all $v \varepsilon V^{\star}$ for which the flat on each side of $q(t)$ at height $p(v)$ is of length two or more (see Figure 1). Notice, by construction, for all veV* this flat must be of length one or more on each side. 


\section{References}

1. P. Champsaur and G. Laroque, Fair allocations in large economies, J. Econ. Theory 25 (1981), 269-282.

2. E.H. Clarke, Multipart pricing of public goods, Public Choice 11 (1971), $17-33$

3. J. Green and J.J. Laffont, Characterization of satisfactory mechanisms for the revelation of preferences for public goods, Econometrica 45 $(1977), 427-438$

4. T. Groves, Incentives in teams, Econometrica 41 (1973), 617-631.

5. T. Groves and J. Ledyard, Some limitations of demand revealing processes, Public Choice 16 (1976), 107-124.

6. T. Groves and M. Loeb, Incentives and public inputs, Journal of Public Economics $4(1975), 211-226$.

7. P. Hammond, Straightforward individual incentive compatibility in large economies, Rev. Econ. Stud. 46 (1979), 263-282.

8. B. Holmström, Groves' scheme on restricted domains, Econometrica 47 (1979), 1137-1144.

9. L. Hurwicz, On informationally decentralized systems, in "Decision and Organization" (C. McGuire and R. Radner, Eds.), North-Holland, Amsterdam, 1972.

10. L. Hurwicz, On allocations attainable through nash equilibria, in "Aggregation and Revelation of Preferences" (J.J. Laffont Ed.), North-Holland, Amsterdam, 1979.

11. L. Hurwicz and M. Walker, "On the Generic Non-optimality of Dominant Strategy Allocation Mechanisms with an Application to Pure Exchange Economies," Dept. of Economics SUNY, Stonybrook, Working Paper No. 250, 1983. 
12. N. Kleinberg, Fair allocations and equal income, J. Econ. Theory 23 $(1980), 189-200$

13. J.-J. Laffont and E. Maskin, "The Theory of Incentives: An Overview," Cambridge University Economic Theory Discussion Paper No. 41, 1981.

14. M. Loeb, Alternative versions of the demand-revealing process, Public Choice $29(1977), 15-26$.

15. L. Makowski, A characterization of perfectly competitive economies with production, J. Econ. Theory 22 (1980), 208-221.

16. L. Makowski and J.M. Ostroy, "Groves Mechanisms in Continuum Economies," UCLA Economics Working Paper, 1986.

17. A. Mas-Cole1l, "On the Second Welfare Theorem for Anonymous Net Trades in Exchange Economies with Many Agents," SEEDS Discussion Paper No. 8, 1982.

18. A. McLennan, Ph.D. Thesis, Princeton University, 1981.

19. J.M. Ostroy, The no-surplus condition as a characterization of perfectly competitive equilibrium, J. Econ. Theory 22 (1980), 183-207.

20. D.J. Roberts and A. Postelwaite, The incentives for price-taking behavior in large exchange economies, Econometrica 44 (1976), 115-127.

21. L. Shapley, On balanced sets and cores, Naval Research Logistics Quarterly 14 (1967), 453-460.

22. W. Vickrey, Counterspeculation, auctions, and competitive sealed tenders, Journal of Finance $16(1961), 8-37$.

23. M. Walker, A note on the characterization of mechanisms for the revelation of preferences, Econometrica 46 (1978), 147-152.

24. M. Walker, On the existence of a dominant stategy mechanism for making optimal public decisions, Econometrica 48 (1980), 1521-1540. 